\title{
SUNCT and optic nerve hypoplasia
}

\author{
Brett J. Theeler $\cdot$ Kevin R. Joseph
}

Received: 16 April 2009/ Accepted: 20 May 2009/Published online: 19 June 2009

(C) Springer-Verlag 2009

\begin{abstract}
SUNCT has been reported in association with abnormalities of the brainstem and pituitary region. We present a patient with a history of left optic nerve hypoplasia, mild hypothalamic-pituitary dysfunction, and SUNCT starting in adolescence. SUNCT with an early age of onset may be associated with congenital abnormality of the hypothalamic-pituitary axis.
\end{abstract}

Keywords SUNCT - Trigeminal autonomic cephalagia Pituitary · Hypothalamus · Optic nerve hypoplasia

\section{Introduction}

Short-lasting unilateral neuralgiform headache attacks with conjunctival injection and tearing (SUNCT) was first described in 1978 with diagnostic criteria established in 2004 [1, 2]. If associated with other cranial autonomic symptoms or without both conjunctival injection and tearing, the syndrome is classified as SUNA (short-lasting unilateral neuralgiform headache attacks with autonomic features) [3]. SUNCT and SUNA are classified as trigeminal autonomic cephalalgias along with cluster headaches, and paroxysmal hemicrania with all of these headache syndromes sharing unilateral headache pain in a V1 trigeminal distribution and ipsilateral activation of cranial

The opinions or assertions contained herein are the private views of the authors and are not to be construed as official or as reflecting the views of the Department of the Army or the Department of Defense.

B. J. Theeler $(\bowtie) \cdot$ K. R. Joseph

Department of Medicine/Neurology Service,

Madigan Army Medical Center,

Fort Lewis, WA, USA

e-mail: btheeler@hotmail.com parasympathetics [2]. SUNCT has been reported in association with acquired and congenital abnormalities of the brainstem and pituitary region [2]. We report a case of SUNCT with onset in adolescence in a patient with unilateral optic nerve hypoplasia $(\mathrm{ONH})$ and mild hypothalamic-pituitary dysfunction.

\section{Case presentation}

A 27-year-old Caucasian woman with a history of left ONH diagnosed before 2 years of age was seen for evaluation of brief head pains. These headaches began at age 14. These head pains occurred over the right orbital region and lasted for around $30 \mathrm{~s}$ and rarely for more than a minute. The pain was described as sharp and severe. The right eye became injected with tearing and right eyelid edema also occurred during the headaches. The headaches would occur three or more times per day for 2-3 days. The patient would have this pattern of headaches 2-3 times per year with no apparent seasonal or diurnal pattern. The attacks appeared to be spontaneous with no clear triggers. The episodes were relatively infrequent but were frightening to the patient and her family. These had been previously considered a manifestation of anxiety.

The patient had a history of abnormal menstrual cycles and galactorrhea starting in adolescence. The patient reported having hyperprolactinemia but prolactin levels from adolescence were not available for our review. In adolescence, she was tried on bromocriptine that did not adequately control her galactorrhea, but she had not had galactorrhea over the preceding 3 years. She has a history of two miscarriages although she completed three natural pregnancies. The patient had elevated prolactin levels of 31.0 and $50.3 \mathrm{ng}$ per milliliter (normal range 3.34-26.72 ng/ml) 
Fig. 1 a Coronal T2 sequence and $\mathbf{b}$ coronal FLAIR sequence showing a small right pituitary cyst and normal septum pellucidum. c Coronal T1 sequence with gadolinium showing a normal pituitary gland and infundibulum. d Coronal orbital inversion recovery sequence showing left optic nerve hypoplasia
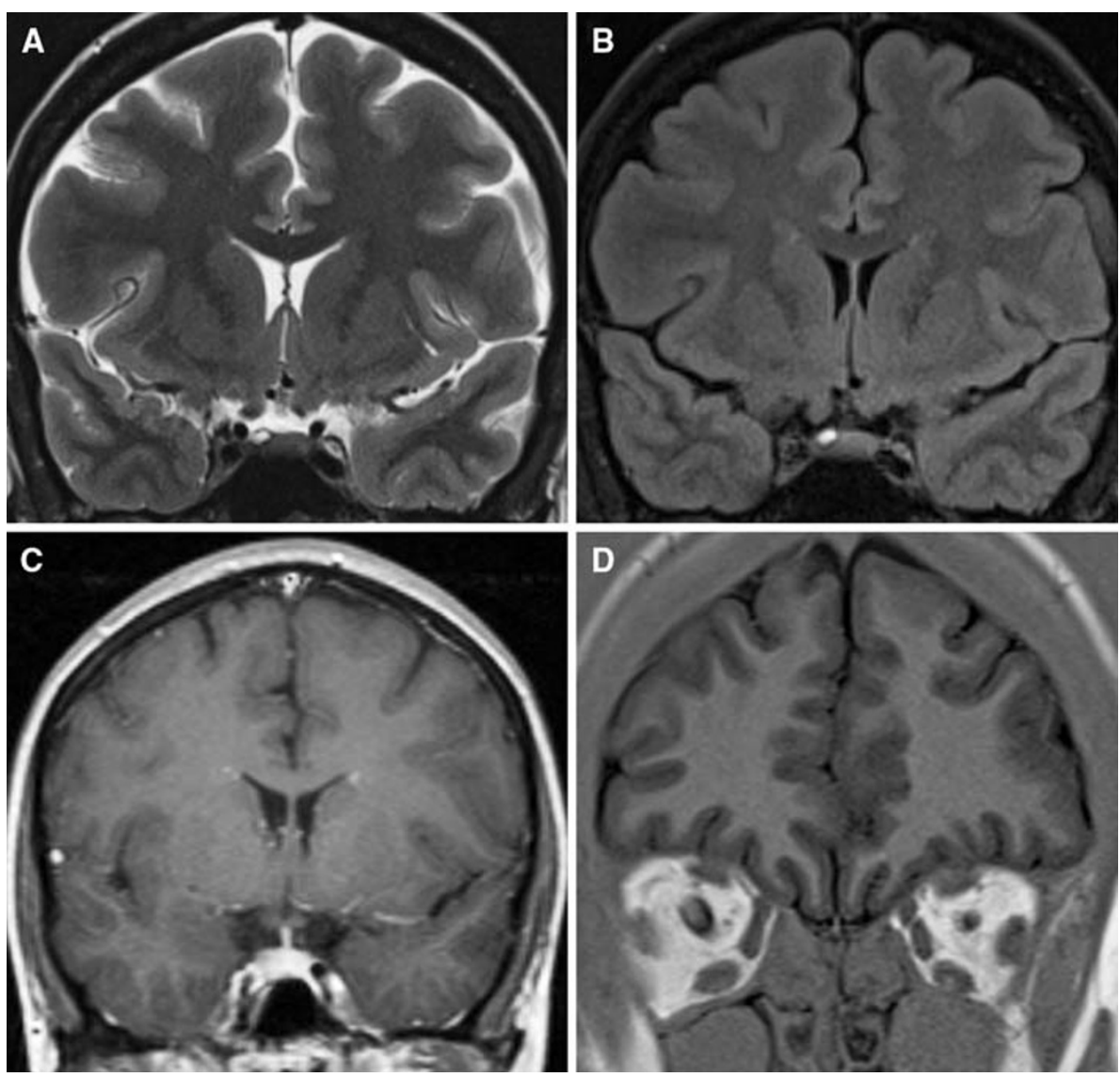

drawn at our facility and a low level of luteinizing hormone 0.76 milli-international units per milliliter (normal range $1.2-103.0 \mathrm{mIU} / \mathrm{ml}$ depending on stage of menstrual cycle) with normal levels of follicle stimulating hormone, renin, aldosterone, growth hormone, insulin-like growth factor-1, adrenocorticotrophic hormone, and thyroid stimulating hormone. Beginning in adolescence, the patient had spells of brief confusion and autonomic symptoms felt to be related to hypoglycemia with finger stick blood glucose levels of 40-50 during or immediately after these spells. The patient had a personal history of simple febrile seizures occurring before age 2 with a family history of simple febrile seizures in her father, twin brother, and in two of her children. There is no other family member with documented ONH. The patient had a normal developmental history and growth velocity throughout childhood and adolescence.

Neurologic examination was remarkable for strabismus and visual function limited to light perception in the left eye. MRI of the Brain with and without contrast revealed a hypoplastic left optic nerve (Fig. 1). The septum pellucidum was present. An MRI of the sellar region with contrast revealed an abnormality that followed the signal of cerebrospinal fluid on T1 and T2 sequences without complete attenuation on FLAIR sequences consistent with a pituitary cyst (Fig. 1). The sellar region otherwise appeared normal and there was no deviation of the infundibulum. A seizure protocol MRI brain did not reveal any abnormalities suggestive of a malformation of cortical development. No abnormalities of the Meckel's cave region or posterior fossa were noted on any of these studies. An electroencephalogram was within normal limits for age.

The patient has been followed for 8 months and continues to have a similar headache pattern. The patient's headaches are infrequent and unpredictable and determining an appropriate treatment strategy has been difficult. The headaches do not occur frequently enough to require preventive therapy. The patient is hesitant to start antiepileptic medications such as topiramate or lamotrigine, so we prescribed indomethacin $25 \mathrm{mg}$ to be used up to three times per day during symptomatic periods with the goal of reducing headache frequency and headache days. The patient has not yet tried the medication.

\section{Discussion}

The patient's headache syndrome met ICHD-2 criteria for short-lasting, unilateral neuralgiform headaches with conjunctival injection and tearing (SUNCT), and was consistent with more recent descriptions of this syndrome [1-3]. This 
patient did not report cutaneous or other triggering factors as seen in many SUNCT patients although spontaneous attacks may be present in $14-28 \%$ of SUNCT patients [3]. The clinical features were not suggestive of short-lasting paroxysmal hemicrania or trigeminal neuralgia. We elected a trial of indomethacin during symptomatic periods. The efficacy of this therapeutic strategy has not yet been determined.

SUNCT has been shown to have increased activation in the hypothalamus ipsilateral to the symptoms and in another case bilaterally on functional MRI [4, 5]. There is also a case of SUNCT responding to hypothalamic stimulation ipsilateral to the symptoms [6] and a case of a SUNCT patient responding to treatment with clomiphene citrate presumably due to its effects on the hypothalamus [7]. There are numerous cases of SUNCT occurring in patients with prolactinomas sometimes during treatment with cabergoline or bromocriptine [8-13]. SUNCT has also been in two patients with congenital bone abnormalities, basilar impression and craniosynostosis, with presumed involvement of the posterior fossa [14]. SUNCT typically has age of onset between 35 and 65 years with a mean around age 50 although ranges of age 10-77 have been reported [2, 3]. SUNCT associated with pituitary or brainstem abnormalities may have a slightly earlier age at onset with a mean around age 43 in one review [14]. The case of craniosynostosis had onset of SUNCT at age 14 similar to the age of onset in our patient suggesting that congenital abnormalities of the posterior fossa or hypothalamic-pituitary axis may precipitate an earlier age of onset [15].

$\mathrm{ONH}$ is bilateral in $80 \%$ of cases [16]. Hypothalamicpituitary dysfunction occurs in $75 \%$ of patients with $\mathrm{ONH}$ and hyperprolactinemia occurs in $62 \%$ of these patients [16]. The pituitary gland is abnormal in only $13 \%$ of patients with $\mathrm{ONH}$ and the septum pellucidum is absent in $38 \%$ of patients [16]. There may be a higher risk of endocrine dysfunction in $\mathrm{ONH}$ patients with an absent septum pellucidum and abnormal pituitary gland on neuroimaging but this is not absolute as patients without these imaging features can still have hypothalamic-pituitary dysfunction. Patients with unilateral ONH are at lower risk of hypothalamic-pituitary dysfunction than those with bilateral disease although dysfunction occurs in $69 \%$ of patient's with unilateral ONH (compared to $81 \%$ with bilateral ONH) [16]. Our patient's chronic hyperprolactinemia, abnormal menstrual cycles, and presumed hypoglycemic episodes were suggestive of mild hypothalamicpituitary dysfunction. We were able to document hyperprolactinemia and low levels of luteinizing hormone, which were the likely associated with her history of abnormal menstrual cycles and galactorrhea. The strong personal and family history of simple febrile seizures may indicate a dysfunction of temperature regulation although another heritable cause cannot be excluded. Problems with body temperature regulation have been reported in $\mathrm{ONH}$, presumably due to hypothalamic dysfunction, with these patients sometimes requiring hospitalizations to rule out infections [16]. Growth hormone deficiency and adrenocorticotrophic hormone deficiency are among the most common endocrinopathies in $\mathrm{ONH}$ patients, both of which can result in hypoglycemia [16]. We were not able to document a deficiency of growth hormone or adrenocorticotrophic hormone to explain the episodes of presumed hypoglycemia. Our patient had a pituitary abnormality on MRI ipsilateral to her symptoms. The imaging characteristics were most consistent with a pituitary cyst and there was no other abnormality of her sellar region. We felt the pituitary cyst was most likely an incidental finding and not the likely cause of her mild hypothalamic dysfunction and SUNCT headaches.

To our knowledge, this is the first reported case of SUNCT in a patient with ONH. We hypothesize that there is an association between SUNCT headaches and the mild, hypothalamic-pituitary dysfunction in our patient. Congenital abnormality of the hypothalamic-pituitary axis may also explain the onset of this headache syndrome in adolescence.

\section{Conflict of interest None.}

\section{References}

1. Headache Classification Subcommittee of the International Headache Society (2004) The international classification of headache disorders, 2nd edn. Cephalalgia 24(Suppl 1):9-160

2. Boes CJ, Swanson JW (2006) Paroxysmal hemicrania, SUNCT, and hemicrania continua. Semin Neurol 26(2):260-270

3. Cohen AS, Matharu MS, Goadsby PJ (2006) Short-lasting unilateral neuralgiform headache attacks with conjunctival injection and tearing (SUNCT) or cranial autonomic features (SUNA) - a prospective clinical study of SUNCT and SUNA. Brain 129(Pt 10):2746-2760

4. May A, Bahra A, Buchel C et al (1999) Functional MRI in spontaneous attacks of SUNCT: short-lasting neuralgiform headache with conjunctival injection and tearing. Ann Neurol 46:791-793

5. Sprenger T, Valet M, Platzer S et al (2005) SUNCT: bilateral hypothalamic activation during headache attacks and resolving of symptoms after trigeminal decompression. Pain 113(3):422-426

6. Leone M, Franzini A, D'Andrea G et al (2005) Deep brain stimulation to relieve drug-resistant SUNCT. Ann Neurol 57(6):924-927

7. Rozen TD, Saper JR, Sheftell FD, Dodick DW (2005) Clomiphene citrate as a new treatment for SUNCT: hormonal manipulation for hypothalamic-influenced trigeminal autonomic cephalalgias. Headache 45(6):754-756

8. Matharu MS, Levy MJ, Merry RT, Goadsby PJ (2003) SUNCT syndrome secondary to prolactinoma. J Neurol Neurosurg Psychiatry 74(11):1590-1592 
9. Massiou H, Launay JM, Levy C et al (2002) SUNCT syndrome in two patients with prolactinomas and bromocriptine-induced attacks. Neurology 58(11):1698-1699

10. Rozen TD (2006) Resolution of SUNCT after removal of a pituitary adenoma in mild acromegaly. Neurology 67(4):724

11. Jiménez Caballero PE (2007) SUNCT syndrome in a patient with prolactinoma and cabergoline-induced attacks. Cephalalgia 27(1):76-78

12. Bosco D, Labate A, Mungari P et al (2007) SUNCT and high nocturnal prolactin levels: some new unusual characteristics. J Headache Pain 8(2):114-118

13. de Lourdes Figuerola M, Bruera O, Pozzo MJ, Leston J (2009) SUNCT syndrome responding absolutely to steroids in two cases with different etiologies. J Headache Pain 10(1):55-57
14. Trucco M, Mainardi F, Maggioni F et al (2004) Chronic paroxysmal hemicrania, hemicrania continua and SUNCT syndrome in association with other pathologies: a review. Cephalalgia 24(3): 173-184

15. Morís G, Ribacoba R, Solar DN, Vidal JA (2001) SUNCT syndrome and seborrheic dermatitis associated with craneosynostosis. Cephalalgia 21:157-159

16. Borchert M, Garcia-Filion P (2008) The syndrome of optic nerve hypoplasia. Curr Neurol Neurosci Rep 8(5):395-403 